\title{
Communication \\ Kinematics and Selection Rules for Light-by-Light Scattering in a Strong Magnetic Field
}

\author{
Anatoly Shabad ${ }^{1,2}$ \\ 1 P. N. Lebedev Physical Institute, 53 Leninskiy prospekt, 119991 Moscow, Russia; shabad@lpi.ru \\ 2 Department of Physics, Tomsk State University, 36 Lenin Prospekt, 634050 Tomsk, Russia
}

Received: 21 August 2020 ; Accepted: 13 November 2020; Published: 17 November 2020

\begin{abstract}
Selection rules that follow from parity and four-momentum conservation are listed for head-on light-by-light scattering in a strong magnetic field taking into account nontrivial dispersion laws of different photon eigen-modes. The wave-length shifts occur for certain transitions between photon eigen-modes.
\end{abstract}

Keywords: photon-photon scattering; strong fields; nonlinear electrodynamics; selection rules

\section{Introduction}

Contrary to the classical electrodynamics of Faraday-Maxwell, the quantum theory of electromagnetic fields, i.e., the theory of the interaction of electrons, positrons, and other charged particles with photons (QED), is nonlinear in the sense that electromagnetic fields are not mutually independent, but they interact between/with themselves. This interaction may become significant provided any of the fields involved are sufficiently strong, the characteristic field-strength scale of nonlinearity being determined by the so-called Schwinger value $\frac{m^{2}}{e}$, where $m$ and $e$ are the mass and charge of the electron. The self-interaction stems from the quantum process of real or the virtual creation of a pair of oppositely charged particles by a photon. Hence, over the time of existing in the intermediate state in the form of charged particles, the photon is interacting with other electromagnetic fields. This is how the effective photon-photon coupling appears.

The nonlinearity of QED has a great number of possible manifestations, an important one among them being the interaction of a photon with a strong external field, its effect being equivalent [1-3] to a sort of anisotropic, linear or nonlinear, homogeneous or inhomogeneous, medium. Photon splitting [2] into two/merging of two photons into one, modification of the Coulomb field [4-6] by an external field, and the magneto-electric effect-the appearance of a magnetic field as produced by a static electric source-in particular, acquiring by this source the feature of being a magnetic dipole [7], are other examples of the effects produced by strong constant field (or a field of an intense laser). Self-interaction yields the electromagnetic renormalization of electric or magnetic moments of nucleons [8]; this is an example that does not require the presence of an external field. Notwithstanding the diversity of manifestations, the nonlinearity is ever reduced ${ }^{1}$, in the end, to the contribution of a single fundamental box diagram of two (real or virtual) photons exchanged by an electron to produce a virtual pair that in its turn annihilates into two photons via exchange by an electron. This four-order diagram is known as light-by-light scattering.

Since QED is a firmly established theory, there is no doubt that the light-by-light scattering, and thereby most of the other nonlinear effects, exists. However, the observation of nonlinearity in

1 except, perhaps, the Schwinger effect of the pair production from the vacuum by a strong constant electric field. 
QED is difficult, because of the extreme smallness, $\sim \alpha^{2}$, of the light-by-light four-point diagram. For this reason, until recently, only a few manifestations of the QED nonlinearity have been reported. These are nonlinear Compton scattering [9] (single-vertex emission of a photon by an electron) and the nonlinear Breit-Wheeler process [10] (single-vertex creation of a pair by a photon). One may add to the list the birefringence evidence in a pulsar [11]. We may speak also about indirect observational evidence of nonlinearity. The photon splitting in a magnetic field [2] has become an organic part of the accepted theory of pulsar radiation (see the review [12]). To a lesser extent, the same relates to the effect of the capture of a gamma-quantum by a curved magnetic field of a pulsar [13]. Promising, but not yet successful, are the endeavored Earth-based measurements of vacuum birefringence. All hopes are placed on the circumstance that the smallness of the fine-structure coupling constant $\alpha$ may be under certain conditions compensated by the strength of the involved fields and by the large energy of the involved particles and the intensity of their fluxes. For instance, a proposed experiment, called LUXE, where a GeV-photon or electron collides with a laser beam, implies that the effective field in the collision area will be up to three times larger than the Schwinger critical field [14]. On the other hand, the necessary conditions are met in the ultraperipheral collisions of protons or heavy highly ionized atoms (especially of lead). The ultrarelativistic charged colliding bodies exchange with two (large intensity beams of) GeV-photons that scatter one another. This process was observed [15] with the LHC in the course of the ATLAS experiment and is viewed as the first experimental evidence of the light-by-light scattering.

Contrary to the reaction of two photons merging into one, forbidden in the vacuum both by the Furry theorem and kinematically on the free-photon mass shell, the four-photon diagram of the two photons scattering into two is absolutely allowed already in the vacuum. In connection with the ATLAS experiment, it becomes interesting to consider this process in the presence of a strong magnetic field, because, as was first noticed by Kharzeev and McLerran [16], once the charged nuclei collide at a nonzero impact parameter, a magnetic field perpendicular to the reaction plane arises; hence, the peripheral interaction takes place in its presence. While the possibility that this magnetic field may open the reaction of two-photon merging under the existing conditions of the ultraperipheral collision is left aside for the time being, the light-by-light scattering is attracting attention.

Recently, R. Baier, A. Rebhan, and M. Wödlinger [17] calculated the cross-sections of the light-by-light scattering as functions of an external magnetic field $\mathbf{B}$ for low-energy long-wave photons, based on the Heisenberg-Euler Lagrangian responsible [18] for that regime. Calculations are done for special cases of equal wave-length photons colliding head-on, when the external field is either parallel or perpendicular to the incoming photon direction, so that the three-momenta of the two colliding photons are subject to the relation $\mathbf{k}_{1}=-\mathbf{k}_{2}$. This kinematical restriction cannot, generally, be avoided by a Lorentz transformation to an arbitrary frame, since-when $\mathbf{B} \nVdash \mathbf{k}_{1,2}$-the external field would be changed by the boost in the direction of the photon propagation. Nevertheless, the head-on collision case corresponds to the realistic experimental situation of the nuclei collision or the collision of two laser beams, so that in the laboratory frame, their center-of-mass is at rest.

We stress the fact that the kinematics of the reaction cannot be taken without changing from the vacuum case, because the presence of the magnetic field affects it. The relation between the energy and momentum of the photon is not just $\omega=|\mathbf{k}|$, but the energy of each photon may depend in two ways on the angle, the direction of the momentum of which makes with the magnetic field, $\omega_{\text {ordinary,extraordinary }}\left(k^{2},(\mathbf{k} \cdot \mathbf{B})^{2}\right)$. This fact reflects the anisotropy of the effective "optical medium" and birefringence ${ }^{2}$ (within the Heisenberg-Euler approximation kept to in [17], and here as well, the dependence on the angle is very simple; see [20]).

2 It is interesting to note that, when the nonlinearity is determined by the Heisenberg-Euler Lagrangian, a traveling-wave solution of nonlinear Maxwell equations is provided by a dispersion law that involves a dependence on the amplitudes of the traveling wave [19]. This is, certainly, not our case, since we deal with the propagation of small-amplitude waves governed by equations linearized near an external field. 
The photons that are inside the magnetic field are classified according to the eigen-modes (if one is willing to consider the scattering of photons coming from outside of the region occupied by the magnetic field, one should also take into account their reflection and refraction at the border). Definite laws of propagation - the corresponding refraction indices, propagation speeds, etc.—are associated with photon eigen-modes, which are not the photons, just transversely polarized (the polarizations of eigen-modes were established in [3] and partially in [2]). For the general angle between $\mathbf{k}$ and $\mathbf{B}$, the so-called ordinary wave is transverse (this is Mode 3 in the classification of [3]), and its electric field is orthogonal to the plane spanned by $\mathbf{k}$ and $\mathbf{B}$, while the extraordinary wave (Mode 2) is not (its electric vector belongs to that plane). Therefore, the scattered photons are not all transverse even when the incoming photons are parallel or orthogonal to the magnetic field.

In the next section, I illustrate the influence of the anisotropic dispersion laws by considering relations among momenta and the scattering angle of the photons within the same configurations of the incident photons and the magnetic fields as the ones considered in [17]. I did not find kinematical bans, analogous to those known for photon splitting, but the bans due to P-conservation like in Adler's work [2] are expected. Essential as well is the change of the photon wave-length depending (for parallel incidence) or not depending (for perpendicular incidence) on the scattering angle.

\section{Kinematics of Scattering a Photon by a Photon}

Since the external field is homogeneous in space and does not depend on time, the energy-momentum conservation reads:

$$
\begin{aligned}
\mathbf{k}_{1}+\mathbf{k}_{2} & =\mathbf{k}_{3}+\mathbf{k}_{4} \\
\omega_{1}+\omega_{2} & =\omega_{3}+\omega_{4} .
\end{aligned}
$$

Each photon $i=1,2,3,4$ may belong to one of polarization modes numbered as $a=1,2,3$. The total number of modes, three, is determined by the dimension of the space. It will be explained later that, at least within the Heisenberg-Euler approximation accepted here, only two modes out of three may correspond to real, propagating electromagnetic waves. Energy in each mode depends on its momentum and on the orientation of the latter with respect to the magnetic field:

$$
\omega_{i}=\omega^{\left(a_{i}\right)}\left(k_{i}^{2},\left(\mathbf{k}_{i} \cdot \mathbf{B}\right)^{2}\right), \quad k_{i}=\left|\mathbf{k}_{i}\right| .
$$

We say that we face the "center-of-mass" configuration if, additionally, the relation:

$$
\mathbf{k}_{1}+\mathbf{k}_{2}=\mathbf{k}_{3}+\mathbf{k}_{4}=0
$$

is obeyed (photons of equal wave-length are colliding head-on). I shall confine myself to the "center-of-mass configuration" (4) in what follows.

With the account of (4), Equation (3) may be written as:

$$
\omega_{i}=\omega^{\left(a_{i}\right)}\left(k_{i}^{2},\left(\mathbf{k}_{i} \cdot \mathbf{B}\right)^{2}\right), \quad k_{1,3}=k_{2,4}, \quad\left(\mathbf{k}_{1,3} \cdot \mathbf{B}\right)^{2}=\left(\mathbf{k}_{2,4} \cdot \mathbf{B}\right)^{2},
$$

i.e., $\mathbf{k}_{1}$ represents the incident photons and $\mathbf{k}_{3}$ represents the scattered ones (When the two incoming photons belong to the same mode, $a_{1}=a_{2}$, the configuration considered may be described as scattering of equal-energy photons, because then, $\omega^{\left(a_{1}\right)}\left(k_{1},\left(\mathbf{k}_{1} \cdot \mathbf{B}\right)^{2}\right)=\omega^{\left(a_{2}\right)}\left(k_{2},\left(\mathbf{k}_{2} \cdot \mathbf{B}\right)^{2}\right)$ thanks to the relation $\mathbf{k}_{1}=-\mathbf{k}_{2}$. I shall not restrict myself to this case in what follows, however.).

\subsection{Eigen-Value Problem for the Polarization Tensor, Polarization, and the Dispersion of Eigen-Modes}

The polarizations and dispersion laws of each mode are obtained via the solution of the eigen-value problem for the $4 \times 4$ polarization tensor $\Pi_{\mu \tau}$ : 


$$
\Pi_{\mu \tau} b^{\tau(a)}=\varkappa_{a} b_{\mu}^{(a)}
$$

The eigen-vectors, which are vector potentials giving polarizations of eigen-modes, were, irrespective of approximation, found in [3] (see also [21] and the author's monograph [22]) to be:

$$
b_{\mu}^{(1)}=\left(F_{\mu \tau}^{2} k^{\tau}\right)\left(\mathbf{k}^{2}-k_{0}^{2}\right)-k_{\mu}\left(k F^{2} k\right), b_{\mu}^{(2)}=\widetilde{F}_{\mu \tau} k^{\tau}, b_{\mu}^{(3)}=F_{\mu \tau} k^{\tau} .
$$

Here, $k_{0}$ is the same as $\omega$; the pseudotensor $\widetilde{F}_{\mu \tau}$ is dual to the field-strength tensor $F_{\mu \tau}$ of the external magnetic field, and $\left(k F^{2} k\right)=k^{\mu} F_{\mu \tau}^{2} k^{\tau}$. The fourth eigen-mode, $b_{\mu}^{(4)}=k_{\mu}, \varkappa_{4}=0$, is physically trivial. The dispersion laws (3) of the three modes are given by the solutions of equations:

$$
\mathbf{k}^{2}-k_{0}^{2}=\varkappa_{a}\left(\mathbf{k}^{2}-k_{0}^{2}, k F^{2} k\right), a=1,2,3 .
$$

These are equations for the photon mass shells to be satisfied by real photons in a magnetic field; if not, photons should be referred to as virtual, even if, generally, $|\mathbf{k}|=\omega$.

\subsubsection{Polarization of Eigen-Modes}

Electric and magnetic fields in the eigen-waves are to be calculated using the eigen-vectors $b_{\mu}^{(a)}$ as four-potentials. In this way, the following orientations of electric fields $e^{(a)}$ are obtained [3], irrespective of whether the photons are off-shell or real:

$$
\begin{aligned}
& \mathbf{e}^{(1)}=-\frac{\mathbf{k}_{\perp}}{k_{\perp}} \omega, \\
& \mathbf{e}_{\perp}^{(2)}=\mathbf{k}_{\perp} k_{\|}, \mathbf{e}_{\|}^{(2)}=\frac{\mathbf{k}_{\|}}{k_{\|}}\left(k_{\|}^{2}-\omega^{2}\right), \\
& \mathbf{e}^{(3)}=-\omega\left(\frac{\mathbf{k}_{\perp}}{k_{\perp}} \times \frac{\mathbf{k}_{\|}}{k_{\|}}\right) .
\end{aligned}
$$

Here, $\mathbf{k}_{\perp}, k_{\|}$are the momentum components, orthogonal and parallel to the magnetic field, respectively. The relation $k F^{2} k=-2 \mathfrak{F} \mathbf{k}_{\perp}^{2}$ takes place.

\subsubsection{Dispersion of Eigen-Modes in the Heisenberg-Euler Approximation}

QED governed in the electromagnetic sector by the Heisenberg-Euler (HE) Lagrangian $\mathfrak{L}$ may be valid only in the long-wave, low-frequency domain, because the space- and time-derivatives of the electromagnetic field strength are excluded from it by the procedure of calculation. The dispersion laws for waves of all three modes in such a local (infrared) approximation are realized as a linear dependence of the energy squared on the momentum components squared [20]:

$$
\omega^{(a)}=\left(k_{\|}^{2}+\mathbf{k}_{\perp}^{2} c^{(a)}\right)^{1 / 2}, a=1,2,3
$$

where the coefficients $c^{(2,3)}$ are dimensionless relativistic-invariant functions of the external field expressed in terms of first- and second-order derivatives ${ }^{3}$ of $\mathfrak{L}$ with respect to the field invariants $\mathfrak{F}=\frac{1}{2}\left(B^{2}-E^{2}\right), \mathfrak{G}=(\mathbf{E} \cdot \mathbf{B})$, on which the relativistic invariant action in fact depends. As for $c^{(1)}$, it is

3 These derivatives are involved, because the polarization tensor $\Pi_{\mu \tau}$ in (6) is defined (see, e.g., [23]) as the second variational derivative of effective action over the vector potentials. 
equal to unity, since $\varkappa_{1}=\left(\mathbf{k}^{2}-k_{0}^{2}\right) \mathfrak{L}_{\mathfrak{F}}$ in the HE approximation according to [20]. It is understood that the derivatives are calculated prior to setting the electric field $\mathbf{E}$ equal to zero.

$$
c^{(1)}=1, c^{(2)}=\frac{1-\mathfrak{L}_{\mathfrak{F}}}{1-\mathfrak{L}_{\mathfrak{F}}+2 \mathfrak{F} \mathfrak{L}_{\mathfrak{G} \mathfrak{G}}}, c^{(3)}=1-\frac{2 \mathfrak{F} \mathfrak{L}_{\mathfrak{F} \mathfrak{F}}}{1-\mathfrak{L}_{\mathfrak{F}}} .
$$

By differentiating the known (see, e.g., [18,24]) HE Lagrangian [25], the quantities involved have the following integral representations as (positive) functions of the dimensionless magnetic field $b=e B / m^{2}$ :

$$
\begin{gathered}
\mathfrak{L}_{\mathfrak{F}}=\frac{\alpha}{2 \pi} \int_{0}^{\infty} \frac{\mathrm{d} t}{t} \exp \left(-\frac{t}{b}\right)\left(\frac{-\operatorname{coth} t}{t}+\frac{1}{\sinh ^{2} t}+\frac{2}{3}\right), \\
2 \mathfrak{F}_{\mathfrak{G} \mathfrak{G}}=\frac{\alpha}{3 \pi} \int_{0}^{\infty} \frac{\mathrm{d} t}{t} \exp \left(-\frac{t}{b}\right)\left(\frac{-3 \operatorname{coth} t}{2 t}+\frac{3}{2 \sinh ^{2} t}+t \operatorname{coth} t\right), \\
2 \mathfrak{F} \mathfrak{L}_{\mathfrak{F} \mathfrak{F}}=\frac{\alpha}{\pi} \int_{0}^{\infty} \frac{\mathrm{d} t}{t} \exp \left(-\frac{t}{b}\right)\left(\frac{\operatorname{coth} t}{2 t}-\frac{t \operatorname{coth} t}{\sinh ^{2} t}+\frac{1}{2 \sinh ^{2} t}\right) .
\end{gathered}
$$

Here, $\alpha=e^{2} / 4 \pi=1 / 137$ is the fine-structure constant. Representations in terms of Hurwitz Zeta functions exist [26], which are more explicit.

It is seen from (13) and (12) that only $\mathbf{k}^{2}-k_{0}^{2}=0$ is the dispersion law for mode $a=1$. Its substitution into $b_{\mu}^{(1)}$ in (7) makes this eigen-vector purely four-longitudinal, $\sim k_{\mu}$, thereby carrying neither an electric, nor a magnetic field. An exception is made by the propagation, parallel to the external magnetic field, since then, the quantity $k F^{2} k=-2 \mathfrak{F} \mathbf{k}_{\perp}^{2}$ also disappears. Now, Mode 1 also comes into play, carrying a transverse electric field, $\mathbf{e}^{(1)} \perp \mathbf{k}$, also orthogonal to the electric field of the ever transverse Mode 3, $\mathbf{e}^{(1)} \perp \mathbf{e}^{(3)}$. However, in that case, mode $a=2$ is excluded, because, according to (10), it becomes a purely longitudinal, $\mathbf{e}^{(2)} \| \mathbf{k}$, electric wave, impossible once $\mathbf{k}^{2}-k_{0}^{2}=k_{\|}^{2}-k_{0}^{2}=0$. This means that simultaneously, only two polarization degrees of freedom are present. We shall take these peculiarities into account when considering the incident photons, parallel to the background field in the next subsections.

The causality requires that the group velocity of the eigen-waves be lesser than or equal to the absolute speed of light $c=1$, while the unitarity requires that the residue of the photon propagator in the pole on the mass shell (12) be positive. These principles yield the convexity conditions ${ }^{4} \mathfrak{L}_{\mathfrak{F} \mathfrak{F}} \geqslant 0$, $\mathfrak{L}_{\mathfrak{G} \mathfrak{G}} \geqslant 0$ [20]. It follows from them, that $c^{(2,3)} \leqslant 1$. These inequalities imply that the dispersion curves lie beyond the light cone $|\omega|=|\mathbf{k}|=0$. While investigating the selection rules, it may be important that $c^{(3)}>c^{(2)}$. This can be demonstrated using ${ }^{5}$ (14)-(16). It is important that the dispersion laws for photons of different modes are not just $\omega^{2}-k^{2}=0$, but they are characterized by nonzero "virtualities":

$$
\left[\omega^{(2,3)}\left(k,(\mathbf{k} \cdot \mathbf{B})^{2}\right)\right]^{2}-\mathbf{k}^{2}=\mathbf{k}_{\perp}^{2}\left[c^{(2,3)}-1\right] \leqslant 0
$$

depending on the momentum component $\mathbf{k}_{\perp}$ transverse to the magnetic field $B$ (to avoid possible misunderstanding, we have to warn that the term "virtuality" may be misleading in our case, since it is nonvanishing for a real photon in a magnetic field, whereas when the "virtuality" disappears, the photon is but virtual). We stress that nonvanishing "virtuality" by no means implies that the

4 These convexity properties are certainly respected by the HE approximation resulting in Equations (15) and (16). The third condition following from the causality principle, $1-\mathfrak{L}_{\mathfrak{F}} \geqslant 0$ can be violated for unrealistic exponentially strong fields $\sim \exp (3 \pi / \alpha)$ (in accord with Equation (30) below) as a manifestation of the intrinsic trouble of QED known as the lack of asymptotic freedom.

5 More generally, the dispersion curve for Mode 3 goes higher than that for Mode $2 \omega^{(3)}\left(k_{i}^{2},\left(k_{i} \cdot B\right)^{2}\right)>\omega^{(2)}\left(k_{i}^{2},\left(k_{i} \cdot B\right)^{2}\right)$, because the lowest energy threshold, where the photon may create an electron-positron pair, is for Mode 3 higher: one particle out of the two created by the photon of this mode belongs to an excited Landau level, whereas the Mode 2 photon may create a pair of particles, both in the ground Landau states. The point is that the polarization tensor is infinite at each of the thresholds; hence, each threshold strongly affects the dispersion law; see [21,22] for further explanations. 
photon is massive: by definition, the mass $M$ is the energy of rest. It is always zero for real photons, $M=\omega(0,0)=0$, due to the gauge invariance.

\subsection{Conservation Laws and Selection Rules}

The energy conservation relations (2):

$$
\omega^{\left(a_{1}\right)}\left(k_{1},\left(\mathbf{k}_{1} \cdot \mathbf{B}\right)^{2}\right)+\omega^{\left(a_{2}\right)}\left(k_{1},\left(\mathbf{k}_{1} \cdot \mathbf{B}\right)^{2}\right)=\omega^{\left(a_{3}\right)}\left(k_{3},\left(\mathbf{k}_{3} \cdot \mathbf{B}\right)^{2}\right)+\omega^{\left(a_{4}\right)}\left(k_{3},\left(\mathbf{k}_{3} \cdot \mathbf{B}\right)^{2}\right)
$$

are fraught with dynamic selection rules that may forbid many of the sixteen transitions (four initial by four final polarization states). Besides, there is the parity ban (cf. [2]) for the transitions with the participation of a total odd number of Mode 2 photons in initial and final states, since the Mode 2 vector-potential, $b_{\mu}^{(2)}=\widetilde{F}_{\mu \tau} k^{\tau}$ in (7) is a pseudovector. Hence, the Mode 2 photon may appear only an even number of times among the four photons participating in the reaction.

\subsubsection{Perpendicular Incidence}

Consider first the simplest case when the incoming photon momenta are perpendicular to the magnetic field, $\mathbf{k}_{1,2} \perp \mathbf{B}$. So are the outgoing momenta, since they lie in the same (reaction) plane. In this case, the longitudinally polarized component of the electric field in the extraordinary Mode 2 wave disappears. Modes 2 and 3 are mutually orthogonal, both transverse, electromagnetic waves. Their dispersion laws are:

$$
\omega_{1,2}=\omega^{\left(a_{1,2}\right)}\left(k_{1}, 0\right), \quad \omega_{3,4}=\omega^{\left(a_{3,4}\right)}\left(k_{3}, 0\right)
$$

The energy conservation relations (2) take the form:

$$
\omega^{\left(a_{1}\right)}\left(k_{1}, 0\right)+\omega^{\left(a_{2}\right)}\left(k_{1}, 0\right)=\omega^{\left(a_{3}\right)}\left(k_{3}, 0\right)+\omega^{\left(a_{4}\right)}\left(k_{3}, 0\right) .
$$

To see what rules these equations imply, let me first consider one out of 16 transitions, when two photons of Mode 2 collide to produce two photons of Mode 3: $(2,2) \rightarrow(3,3)$. Equation (19) requires:

$$
\omega^{(2)}\left(k_{1}, 0\right)=\omega^{(3)}\left(k_{3}, 0\right)
$$

or using (12):

$$
k_{1}\left(c^{(2)}\right)^{1 / 2}=k_{3}\left(c^{(3)}\right)^{1 / 2} .
$$

This relation establishes a mandatory connection between the wave-lengths of the incoming and outgoing photons. Since $c^{(3)}>c^{(2)}$, the outgoing wave has a longer length, $k_{3}<k_{1}$. For the opposite process $(3,3) \rightarrow(2,2)$, the selection rule:

$$
k_{1}\left(c^{(3)}\right)^{1 / 2}=k_{3}\left(c^{(2)}\right)^{1 / 2}
$$

implies the opposite inequality: $k_{1}<k_{3}$.

On the contrary, the transitions when two different mode photons turn into also two different mode photons, $(3,2) \rightarrow(3,2)$, demand that $k_{1}=k_{3}$, because Equation (19) becomes in this case:

$$
k_{1}\left(c^{(2)}\right)^{1 / 2}+k_{1}\left(c^{(3)}\right)^{1 / 2}=k_{3}\left(c^{(2)}\right)^{1 / 2}+k_{3}\left(c^{(3)}\right)^{1 / 2} .
$$

It remains to consider transitions when all four photons are of the same polarization, $(2,2) \rightarrow(2,2)$ and $(3,3) \rightarrow(3,3)$ :

$$
\omega^{(2,3)}\left(k_{1}, 0\right)+\omega^{(2,3)}\left(k_{1}, 0\right)=\omega^{(2,3)}\left(k_{3}, 0\right)+\omega^{(2,3)}\left(k_{3}, 0\right) .
$$




$$
k_{1}\left(c^{(2,3)}\right)^{1 / 2}+k_{1}\left(c^{(2,3)}\right)^{1 / 2}=k_{3}\left(c^{(2,3)}\right)^{1 / 2}+k_{3}\left(c^{(2,3)}\right)^{1 / 2} .
$$

Therefore, such a process requires, as before, that $k_{1}=k_{3}$.

Other transitions $(2,2) \leftrightarrow(2,3),(3,3) \leftrightarrow(2,3)$ are forbidden since they would violate parity.

To conclude this subsection, we state that the light-by-light scattering in the "center-of-mass" configuration across the magnetic field requires that the wave-length should be conserved, $k_{1}=k_{3}$, when none or one photon changes its polarization, but it should not, $k_{1} \gtrless k_{3}$, when two photons both change their polarization. Processes, where the photon, whose polarization is given by Mode 2, is involved once or thrice are parity-impossible.

\subsubsection{Parallel Incidence}

When two initial photons are oriented parallel to B (and the final are not), the energy conservation (2) gives:

$$
\omega^{\left(a_{1}\right)}\left(k_{1}, k_{1}^{2} B^{2}\right)+\omega^{\left(a_{2}\right)}\left(k_{1}, k_{1}^{2} B^{2}\right)=\omega^{\left(a_{3}\right)}\left(k_{3},\left(\mathbf{k}_{3} \cdot \mathbf{B}\right)^{2}\right)+\omega^{\left(a_{4}\right)}\left(k_{3},\left(\mathbf{k}_{3} \cdot \mathbf{B}\right)^{2}\right) .
$$

The incident photons may belong either to (transverse in this case) Mode 1 or to ever transverse Mode 3, since Mode 2 disappears for parallel propagation on the mass shell (12) (see (10) with $\left.\mathbf{k}_{\perp}=0,\left(k_{\|}^{2}-\omega^{2}\right)=0\right)$. On the other hand, restricting ourselves to non-forward scattering and bearing in mind that Mode 1 exists on-shell only for parallel propagation, we do not admit the appearance of Mode 1 in the final state, whereas Mode 2 is admitted.

Let all four involved photons belong to Mode $3: a_{1,2,3,4}=3$, that is we consider the process $(3,3) \rightarrow(3,3)$. Then, $(23)$ becomes:

$$
\left.\omega^{(3)}\left(k_{1}, k_{1}^{2} B^{2}\right)=\omega^{(3)}\left(k_{3}, k_{3}^{2}(B \cos \theta)^{2}\right)\right) .
$$

Here, $\theta$ is the scattering angle, of Equation (14) in [17], i.e., the angle the outgoing photon makes with the magnetic field. With the help of (12), we obtain for (24):

$$
\begin{aligned}
& k_{1}=\left(k_{3}^{2}(\cos \theta)^{2}+\left(k_{3}^{2}-k_{3}^{2}(\cos \theta)^{2}\right) c^{(3)}\right)^{1 / 2} \\
& k_{1}=k_{3}\left(\cos ^{2} \theta+c^{(3)} \sin ^{2} \theta\right)^{1 / 2}
\end{aligned}
$$

This is a definite kinematical relation between the scattering angle and the ratio of the initial and final wave-lengths parameterized by the magnetic field hidden in $c^{(3)}$, mandatory for the chosen process to be permitted. Here, the present case of parallel incidence differs from the perpendicular incidence of the previous item, where this ratio was fixed, but the scattering angle remained unrestricted. We shall see below in this item that the same feature is characteristic for all the remaining cases of parallel incidence as well.

It follows from $(25)$ and the causality $c^{(3)} \leqslant 1$, that $k_{1} \leqslant k_{3}$, the outgoing waves are shorter than the incoming ones.

Let now two incident photons belong both to Mode 3, $a_{1,2}=3$, while the scattered photons to Mode $2: a_{3,4}=2$. Then, for the process $(3,3) \rightarrow(2,2),(23)$ becomes:

$$
\begin{gathered}
\left.\omega^{(3)}\left(k_{1}, k_{1}^{2} B^{2}\right)=\omega^{(2)}\left(k_{3}, k_{3}^{2}(B \cos \theta)^{2}\right)\right) . \\
k_{1}=k_{3}\left(\cos ^{2} \theta+c^{(2)} \sin ^{2} \theta\right)^{1 / 2}, k_{1} \leqslant k_{3} .
\end{gathered}
$$

This relation is of the same type as (25).

More special are the cases where one or both incident photons belong to Mode 1 . These are $(1,3) \rightarrow(2,2),(1,3) \rightarrow(3,3),(1,1) \rightarrow(3,3),(1,1) \rightarrow(2,2)$. Let us take the process $(1,3) \rightarrow(2,2)$ to 
begin with. The dispersion law for Mode 1 under parallel propagation is just the vacuum dispersion law $\omega^{(1)}\left(k, k^{2} B^{2}\right)=k$. The energy conservation reads:

$$
\begin{gathered}
\left.\left.\omega^{(1)}\left(k_{1}, k_{1}^{2} B^{2}\right)+\omega^{(3)}\left(k_{1}, k_{1}^{2} B^{2}\right)=\omega^{(3)}\left(k_{3}, k_{3}^{2}(B \cos \theta)^{2}\right)\right)+\omega^{(2)}\left(k_{3}, k_{3}^{2}(B \cos \theta)^{2}\right)\right) . \\
k_{1}\left(1+\left(\cos ^{2} \theta+c^{(3)} \sin ^{2} \theta\right)^{1 / 2}\right)= \\
k_{3}\left(\left(\cos ^{2} \theta+c^{(2)} \sin ^{2} \theta\right)^{1 / 2}+\left(\cos ^{2} \theta+c^{(2)} \sin ^{2} \theta\right)^{1 / 2}\right), k_{1} \leqslant k_{3} .
\end{gathered}
$$

The concluding inequality is established by the term-by-term comparison of the left- and right-hand parts.

The result for the process $(1,3) \rightarrow(3,3)$ is obtained by changing the superscript (2) to (3). Again $k_{1} \leqslant k_{3}$.

Analogously, the process $(1,1) \rightarrow(3,3)$ requires that:

$$
k_{1}=k_{3}\left(\cos ^{2} \theta+c^{(3)} \sin ^{2} \theta\right)^{1 / 2}, \quad k_{1} \leqslant k_{3}
$$

and the process $(1,1) \rightarrow(2,2)$ that:

$$
k_{1}=k_{3}\left(\cos ^{2} \theta+c^{(2)} \sin ^{2} \theta\right)^{1 / 2}, \quad k_{1} \leqslant k_{3} .
$$

This is the same as (26). The remark below Equation (25) relates to Equations (26)-(29) as well.

To conclude, I state that for every case of parallel incidence the outcoming photons have shorter wave-lengths, the two outgoing photons belong to the same mode, and the relation between momenta is (25) when this mode is 3 , and (26) when this mode is 2 . The only exception from this rule is the more complicated relation given by (27).

\subsection{Quantitative Side of the Wave-Length Shifts}

Let the magnetic field essentially exceed the characteristic value $B_{c r}=\frac{m^{2}}{e}=4.4 \cdot 10^{13} \mathrm{G}$, that is let $b=\frac{B}{B_{c r}} \gg 1$. This is expected to occur under the collision of heavy ions. Then, it becomes possible to establish especially simple explicit dependence on the magnetic field of the coefficients $c^{(2,3)}$ that describe the differences between the wave-lengths of the incoming and outgoing photons as given in the previous subsection. Referring to the known asymptotic behavior of the functions (14)-(16) [20]:

$$
\mathfrak{L}_{\mathfrak{F}} \simeq \frac{\alpha}{3 \pi} \ln b, \quad 2 \mathfrak{F} \mathfrak{L}_{\mathfrak{G} \mathfrak{G}} \simeq \frac{\alpha}{3 \pi} b, \quad 2 \mathfrak{F} \mathfrak{L}_{\mathfrak{F} \mathfrak{F}} \simeq \frac{\alpha}{3 \pi},
$$

we can set:

$$
c^{(2)}=\left(1+\frac{\alpha}{3 \pi} b\right)^{-1}, c^{(3)}=1
$$

in the large-field regime $b \gg 1$.

The "virtuality" (17) for Mode 2:

$$
\left[\omega^{(2)}\right]^{2}-\mathbf{k}^{2}=-\mathbf{k}_{\perp}^{2} \frac{\frac{\alpha}{3 \pi} b}{1+\frac{\alpha}{3 \pi} b}
$$

essentially differs from zero for large fields and cannot be ignored when calculating the probabilities of the light-by-light scattering. 


\subsubsection{Perpendicular Incidence}

With the Equation (31), the wave-length shift, Equations (20) and (21), respectively, reads:

$$
k_{1}\left(1+\frac{\alpha}{3 \pi} b\right)^{-1 / 2}=k_{3}, k_{1}=k_{3}\left(1+\frac{\alpha}{3 \pi} b\right)^{-1 / 2} ;
$$

hence, the ratio between the wave-lengths $\left(1+\frac{\alpha}{3 \pi} b\right)^{ \pm 1 / 2}$ differs considerably from unity as $b$ ranges from $10^{2}$ to $10^{3}$ (note that $\frac{\alpha}{3 \pi}=0.77 \cdot 10^{-3}$ ) for the reactions $(2,2) \longleftrightarrow(3,3)$ in perpendicular incidence. Recall that in the previous subsection, it was shown that for perpendicular incidence in the rest of parity-permitted reactions $(3,2) \longleftrightarrow(3,2),(2,2) \longleftrightarrow(2,2),(3,3) \longleftrightarrow(3,3)$, the wave-length remains intact irrespective of the strength of the magnetic field.

\subsubsection{Parallel Incidence}

For parallel incidence, the relation (26) serving the process $(3,3) \rightarrow(2,2)$ and the same relation (29) serving the process $(1,1) \rightarrow(2,2)$ become:

$$
k_{1}=k_{3}\left(\frac{1+\frac{\alpha b}{3 \pi} \cos ^{2} \theta}{1+\frac{\alpha b}{3 \pi}}\right)^{1 / 2} .
$$

The difference between the wave-lengths is most pronounced for the scattering to the angle $\theta=\pi / 2$. The energy ratio between the outgoing and incoming photons is

$$
\left(\frac{\omega_{3}}{\omega_{1}}\right)^{2}=1+\frac{\sin ^{2} \theta}{1+\frac{\alpha b}{3 \pi} \cos ^{2} \theta} \geqslant 1 .
$$

For the process $(1,3) \rightarrow(2,2)$ under parallel incidence, Equation (27) becomes a relation very similar to the previous one

$$
2 k_{1}=k_{3}\left(1+\left(\frac{1+\frac{\alpha b}{3 \pi} \cos ^{2} \theta}{1+\frac{\alpha b}{3 \pi}}\right)^{1 / 2}\right)
$$

Again, the wave-length shift is best pronounced for the scattering angle $\theta=\pi / 2$.

For the process $(1,3) \rightarrow(2,3)$ under parallel incidence, Equation (27) becomes a relation very similar to the previous one:

$$
2 k_{1}=k_{3}\left(1+\left(\frac{1+\frac{\alpha b}{3 \pi} \cos ^{2} \theta}{1+\frac{\alpha b}{3 \pi}}\right)^{1 / 2}\right)
$$

Again, the wave-length shift is best pronounced for the scattering angle $\theta=\pi / 2$.

For other processes under parallel incidence, the wave-length shift is negligible.

\section{Conclusions}

We established selection rules for the head-on photon-photon collisions in the vacuum filled by a strong magnetic field, which is parallel or perpendicular to the axis, on which the momenta of the two incoming photons lie. Some combinations of the initial and final photon polarization eigen-states proved to be excluded by the parity conservation. All the rest are kinematically permitted, but the wave-lengths of the final photons for certain combinations of initial and final polarizations differ from those of the initial photons. This difference may become significant for the supercritical magnetic fields above $B_{c r}=\frac{m^{2}}{e}=4.4 \cdot 10^{13} \mathrm{G}$. This means, in particular, that the device registering the photons resulting from two photon beams' collision should be tuned to an energy different from the energy of the colliding photons. This prescription results from taking into account different dispersion laws 
in different photon modes. The quantitative part of the consideration fits low-frequency, long-wave photons because it is based on the local approximation as given by the HE Lagrangian.

Funding: This research received no external funding.

Acknowledgments: Supported by the RFBR under Project 20-02-00193 and by the TSU Competitiveness Improvement Program, by a grant from "The Tomsk State University D. I. Mendeleev Foundation Program".

Conflicts of Interest: The author declares no conflict of interest.

\section{References}

1. Erber, T. High-energy electromagnetic conversion processes in intense magnetic fields. Rev. Mod. Phys. 1966, 38, 626. [CrossRef]

2. Adler, S. Photon splitting and photon dispersion in a strong magnetic field. Ann. Phys. 1971, 67, 599-647. [CrossRef]

3. Batalin, I.A.; Shabad, A.E. Photon Green Function in a Stationary Homogeneous Field of the Most General Form. Sov. Phys. JETP 1971, 33, 483.

4. Loskutov, Y.M.; Skobelev, V.V. Nonlinear electrodynamics in a superstrong magnetic field. Phys. Lett. A 1976, 56, 151. [CrossRef]

5. Shabad, A.E.; Usov, V.V. Modified Coulomb law in a strongly magnetized vacuum. Phys. Rev. Lett. 2007, 98, 180403. [CrossRef] [PubMed]

6. Sadooghi, N.; Jalili, A.S. New look at the modified Coulomb potential in a strong magnetic field. Phys. Rev. D 2007, 76, 065013. [CrossRef]

7. Gitman, D.M.; Shabad, A.E. Nonlinear (magnetic) correction to the field of a static charge in an external field. Phys. Rev. D 2012, 86, 125028. [CrossRef]

8. Costa, C.V.; Gitman, D.M.; Shabad, A.E. Nonlinear corrections in basic problems of electro-and magneto-statics in the vacuum. Phys. Rev. D 2013, 88, 085026. [CrossRef]

9. Burke, D.L.; Field, R.C.; Horton-Smith, G.; Spencer, J.E.; Walz, D.; Berridge, S.C.; Bugg, W.M.; Shmakov, K.; Weidemann, A.W.; Bula, C.; et al. Positron production in multi-Photon light by light scattering. Phys. Rev. Lett. 1997, 79, 1626-1629. [CrossRef]

10. Brabec, T. Strong Field Laser Physics; Springer Series on Optical Sciences; Springer: Berlin/Heidelberg, Germany, 2009; Volume 134.

11. Mignani, R.P.; Testa, V.; Caniulef, D.G.; Taverna, R.; Turolla, R.; Zane, S.; Wu, K. Evidence for vacuum birefringence from the first optical polarimetry measurement of the isolated neutron star RX J1856. Mon. Not. R. Astron. Soc. 2017, 465, 492. [CrossRef]

12. Lai, D.; Salpeter, E.E. Motion and ionization equilibrium of hydrogen atoms in a superstrong magnetic field. Phys. Rev. A 1995, 52, 2611. [CrossRef] [PubMed]

13. Shabad, A.E.; Usov, V.V. Gamma-quanta capture by magnetic field and pair creation suppression in pulsars. Nature 1982, 295, 215. [CrossRef]

14. Abramowicz, H.; Altarelli, H.; Aßmann, M.R.; Behnke, T.; Benhammou, Y.; Borysov, O.; Borysova, M.; Brinkmann, R.; Burkart, F.; Büßer, K.; et al. Letter of intent for the LUXE experiment. arXiv 2019, arXiv:1909.00860.

15. Aaboud, M.; Burger, N.; Delmastro, A.M.; Ciaccio, M.D.; Elles, L.S. Evidence for light-by-light scattering in heavy-ion collisions with the ATLAS detector at the LHC. Nat. Phys. 2017, 13, 852-858. [CrossRef]

16. Kharzeev, D.E.; McLerran, L.D.; Warringa, H.J. The Effects of topological charge change in heavy ion collisions: Event by event $\mathrm{P}$ and CP violation. Nucl. Phys. A 2008, 803, 227-253. [CrossRef]

17. Baier, R.; Rebhan, A.; Wödlinger, M. Light-by-light scattering in the presence of magnetic fields. Phys. Rev. D 2018, 98, 056001. [CrossRef]

18. Berestetsky, V.B.; Lifshitz, E.M.; Pitayevsky, L.P. Quantum Electrodynamics (Nauka, Moscow, 1989); Pergamon Press: Oxford, UK, 1982.

19. Manjarres, A.D.B.; Nowakowski, M. Travelling waves in the Heisenberg-Euler electrodynamics. Phys. Rev. A 2017, 95, 043820. [CrossRef]

20. Shabad, A.E.; Usov, V.V. Effective Lagrangian in nonlinear electrodynamics and its properties of causality and unitarity. Phys. Rev. D 2011, 83, 105006. [CrossRef] 
21. Shabad, A.E. Photon dispersion in a strong magnetic field. Ann. Phys. 1975, 90, 166. [CrossRef]

22. Shabad, A.E. Polarization of the Vacuum and a Quantum Relativistic Gas in an External Field; Nova Science Publishers: New York, NY, USA, 1991.

23. Weinberg, S. The Quantum Theory of Fields; University Press: Cambridge, UK, 2001.

24. Dittrich, W.; Reuter, M. Effective Lagrangians in Quantum Electrodynamics; Lecture Notes in Physics; Springer: Berlin/Heidelberg, Germany, 1985; Volume 220.

25. Heisenberg, W.; Euler, H. Consequences of Dirac's Theory of the Positron. Z. Phys. 1936, 98, 714. [CrossRef]

26. Karbstein, F.; Shaisultanov, R. Photon propagation in slowly varying inhomogeneous electromagnetic fields. Phys. Rev. D 2015, 91, 085027. [CrossRef]

Publisher's Note: MDPI stays neutral with regard to jurisdictional claims in published maps and institutional affiliations.

(C) 2020 by the author. Licensee MDPI, Basel, Switzerland. This article is an open access article distributed under the terms and conditions of the Creative Commons Attribution (CC BY) license (http:// creativecommons.org/licenses/by/4.0/). 